\begin{abstract}
Limited research has examined distress or help-seeking knowledge and attitudes among international students. The aim of this study was to examine psychological distress, mental health literacy (MHL), and help-seeking attitudes and intentions among a sample of domestic and international tertiary students. The sample comprised 357 domestic and international tertiary students. Participants completed an online questionnaire package, with measures relating to distress, MHL, help-seeking attitudes, and help-seeking intentions. International student status was predictive of lower scores on MHL, help-seeking attitudes, and helpseeking intentions for suicidal ideation. Student group was not predictive of help-seeking intentions for emotional problems or psychological distress, but international students had lower help-seeking intentions for suicidal ideation. Educational interventions targeting MHL and help-seeking attitudes and intentions among international students may be required to improve help-seeking behaviours.
\end{abstract}

Declaration of Interest: None to declare.

Keywords: international students; tertiary education; mental health literacy; help-seeking; psychological distress 


\section{A Comparison of Mental Health Literacy, Attitudes, and Help-seeking Intentions among Domestic and International Tertiary Students}

Many studies have documented the various psychosocial, academic, and financial challenges in the university environment (Adlaf, Gliksman, Demers, \& Newton-Taylor, 2001; Furnham, Cook, Martin, \& Batey, 2011; Palmer and Puri, 2006; Ryan, Shochet, \& Stallman, 2010). Such challenges appear more marked for international students, due to the additional stressors of transitioning to a new culture and social and learning environment (Khawaja and Dempsey, 2007; Khawaja and Dempsey, 2008; Lu, Dear, Johnston, Wootton, \& Titov, 2013; Mori, 2000; Reavley and Jorm, 2010). There have been many terms in the literature developed to explain the adjustment difficulties of international tertiary students, including “foreign student syndrome” (Ward, 1967), "acculturative stress" (Berry, Kim, Minde, \& Mok, 1987), and "uprooting disorder" (Tsang, 2001). A number of studies report that international students experience high levels of psychological distress, including helplessness, social withdrawal, loneliness, homesickness, irritability, and even hostility (Sandhu, 1995; Sawir, Marginson, Deumert, Nyland, \& Ramia, 2008). An important aspect of understanding these mental health needs is examining whether these difficulties are unique to the experience of being an international student and whether there are differences between international and domestic students in understanding of and attitudes toward mental health problems.

A number of theories have been proposed to understand the experiences of international students. Broadly, contemporary theories have focused on the cognitive, social and behavioural, and affective adaptation of the individual to their new environment (Zhou, Jindal-Snape, Topping, \& Todman, 2008). For example, social identification theories typically focus on the cognitive changes associated with cultural adaptation, which may include changes to one's self-identity (e.g., Deaux, 1996). This change process may be 
associated with increased mental health concerns. However, across these theories, the links between adaptation and psychological functioning require further research. For example, successful adaptation may not necessarily lead to better psychological functioning if the individual still feels guilt or loss for the transition. There is a need for greater research examining the psychological processes, including reactions to distress (e.g., help-seeking intentions and attitudes), among international students.

\section{Psychological Distress among University Students}

Psychological distress is common among university students (Cooke, Bewick, Barkham, Bradley, \& Audin, 2006; Khawaja and Dempsey, 2007; Lu, et al., 2013; Stallman, 2010). However, despite substantial research conducted on the psychological well-being of university students, relatively little research has been carried out on the psychological wellbeing and distress levels of international students (Khawaja and Dempsey, 2007; Khawaja and Dempsey, 2008; Lu, et al., 2013). Previous research has found that when compared to domestic students, international students have higher psychiatric morbidity rates (Janca and Hetzer, 1992) and experience additional difficulties unique to their situation (Toyokawa and Toyokawa, 2002).

A number of studies have compared psychological wellbeing, distress, or psychiatric morbidity between international and domestic students, with results largely mixed as to whether differences exist between the two groups on indicators of mental health (e.g., Fritz, Chin, \& DeMarinis, 2008; Janca and Hetzer, 1992). To the authors' knowledge, only one study has been conducted in the Australian context comparing the psychological well-being of domestic and international tertiary students. Khawaja and Dempsey (2008) compared the two groups of university students on psychological variables such as psychological distress, social support, mismatched expectations, academic stress, and dysfunctional coping strategies. When compared to domestic students, international students had higher 
mismatched expectations of university life with their actual experience, had less perceived social support, and utilised more dysfunctional coping strategies. However, contrary to hypotheses, international students reported similar levels of psychological distress in comparison with domestic students. Overall, this study highlighted that the distress was equivalent between the two student populations but international students tended to have lower levels of social support and poorer coping mechanisms to deal with this distress when compared with their domestic counterparts. The international students were more likely to adopt coping strategies such as avoidance and repression of their difficulties instead of accessing suitable services. However, help-seeking intentions and factors that influence these intentions were not examined.

\section{Help-seeking Behaviours among University Students}

Despite evidence suggesting a higher level of mental health problems among university students, the utilisation of mental health services by this population is relatively low. Among an American sample, up to $84 \%$ of university students who screened positive for anxiety or depression had not accessed treatment services for the disorder (Eisenberg, Golberstein, \& Gollust, 2007). In a survey of 1,168 students across three Australian universities, Stallman and Shochet (2009) found that $83.9 \%$ of students experienced elevated psychological distress, out of which only $34.3 \%$ had engaged in help-seeking behaviours. Similar to the general tertiary student population, there is an observable trend of underutilisation of mental health services among international student populations (Morgan, Ness, \& Robinson, 2003; Nilsson, Berkel, Flores, \& Lucas, 2004; Raunic and Xenos, 2008; Yakushko, Davidson, \& Sanford-Martens, 2008). In particular, a survey of Chinese-speaking international students in Australia identified that $54 \%$ of the students reported high psychological distress, with only $9 \%$ engaging with a mental health service for assistance (Lu, et al., 2013). In addition to low help-seeking rates among domestic and international 
students, a number of studies have examined differences in help-seeking rates between the two student populations and found that international students demonstrate lower service use compared to their counterparts (Hunt and Eisenberg, 2010; Khawaja and Stallman, 2011; Morgan, et al., 2003; Nilsson, et al., 2004). Despite these differences, there remains a lack of research in this population examining factors that may be influencing such disparities in helpseeking intentions.

\section{Mental Health Literacy}

A factor that has been widely discussed as a facilitator of help-seeking is mental health literacy. Jorm et al. (1997) coined the term "Mental Health Literacy (MHL)" which can be defined as "knowledge and beliefs about mental disorders which aid their recognition, management or prevention" (p.182). A number of studies have indicated that levels of knowledge about mental health and available help-seeking options is less than optimal in the university student community, and particularly so within the international student community (Ang and Liamputtong, 2008; Lu, et al., 2013; Raunic and Xenos, 2008; Reavley, McCann, \& Jorm, 2012; Shea and Yeh, 2008). In the previously mentioned survey of Chinese-speaking international students attending an Australian university, common barriers to seeking professional help for mental health problems were reported as being limited knowledge of available mental health services, the perception that their distress was not severe enough to access treatment, and the lack of knowledge of symptoms of psychological distress (Lu, et al., 2013). However, much of this research has been descriptive or qualitative in nature. There is a need for more precise measurement of MHL in this field, using quantitative and psychometrically robust methods (O'Connor and Casey, 2015; O’Connor, Casey, \& Clough, 2014).

\section{The Current Study}


Given the increasing numbers of individuals choosing to study abroad, it is vital to understand their psychological well-being while at university. The purpose of the current study was to examine potential differences in mental health and related constructs, such as MHL and help-seeking attitudes, between domestic and international students in Australia. Though this study was primarily exploratory in nature, based on the prior studies reviewed above we expected to observe lower MHL and poorer help-seeking attitudes and intentions among international students. Furthermore, based on recent examination of mental health differences between domestic and international students in an Australian context (Khawaja and Dempsey, 2008), we expected to observe similar overall levels of psychological distress between the two student groups.

\section{Method}

\section{Participants}

A power analysis was conducted using G*Power 3.1 (Faul, Erdfelder, Buchner, \& Lang, 2009) to determine the minimum sample size required for the between-groups analysis. The power analysis indicated that 128 participants (64 in each group) were required, based on a power level of .80 , and an estimated medium effect size $(d=.5)$, when employing the traditional .05 criterion for statistical significance.

Data were obtained from a total of 357 domestic and international students from an Australian university and associated tertiary college. These students included undergraduate, post-graduate, and tertiary preparation (such as diploma) students across all study areas. The sample comprised 148 domestic students and 209 international students. Among the domestic students, 26 were male and 122 female, with ages ranging between 17 and 59 years $(M=$ 25.5, $S D=9.46)$. Among the international students, 79 were male and 130 were female, with ages ranging between 17 and 52 years $(M=23.0, S D=5.47)$. The characteristics of the two student populations are further explored as part of the main statistical analyses. 


\section{Design}

The study was a between-groups (two levels: international or domestic) crosssectional survey design. Data were examined in an exploratory fashion for potential differences between groups on the dependent variables of psychological distress, MHL, and help-seeking attitudes and intentions.

\section{Materials and Measures}

Demographics. All participants were asked to provided demographic information, including: age, gender, student status (i.e. international student or not), ethnicity, length of time lived in Australia, educational qualifications or current degree, years of university completed, and previous use of mental health services (MHSs).

General psychological distress. The Kessler-10 (K-10; Kessler et al., 2003) is a 10item questionnaire that provides a global measure of psychological distress based on questions about the level of anxiety and depressive symptoms in the most recent four-week period. Total scores range from $10-50$, with higher scores indicating higher levels of psychological distress. Internal consistency in the current study was high $(\alpha=.91)$.

Help-seeking attitudes. Attitudes towards help-seeking were assessed using the Inventory of Attitudes toward Mental Health Services (IAMHS; Mackenzie, Knox, Gekoski, \& Macaulay, 2004). The 24-item measure has three subscales, psychological openness, helpseeking propensity, and indifference to stigma. In the current study, the full scale demonstrated high internal consistency (Cronbach's alpha coefficient $=.86$ ).

Help-seeking intentions. Intentions to seek help for mental health problems were measured by the General Help-Seeking Questionnaire (GHSQ; Wilson, Deane, \& Ciarrochi, 2005). The scale consists of two subscales, one for each type of problem: suicidal problems and personal-emotional problems. A higher score indicates greater intentions to seek help. Internal consistency in the current study was acceptable for the emotional problems scale $(\alpha$ 
$=.72$ ) and for the suicidal problems scale $(\alpha=.77)$. In the current study, as intentions to seek professional help were of primary interest, a variable labelled highest help-seeking intention, was created for both suicidal and emotional problem subscales. Each participant was allocated the highest score they gave to any one professional help source from the three included in the measure (mental health professional, phone helpline, doctor/GP). As described in Smith and Shochet (2011), this scoring method is preferable to an average score of the professional sources as an individual need only be willing to seek help from one particular professional source in order to receive appropriate assistance.

Mental health literacy. MHL was assessed using the Mental Health Literacy Scale (O’Connor and Casey, 2015), which measures knowledge about mental health and related issues. Responses summed to produce a total score, with higher scores indicating greater MHL. In the current study internal consistency was high (Cronbach's alpha $=.92)$.

\section{Procedure}

Ethical approval was granted from the university's human research ethics committee. Participants were recruited by posters placed across the university campus, posts on social networking sites (e.g. Facebook), and recruitment emails sent to all students through the university's internal email system. As an incentive, all participants were entered into a draw to win one of four $\$ 50$ shopping gift cards for participation. Additionally, first year psychology student participants (13.45\% of sample) received 1-hour course credit for participation. Consent, demographics, and questionnaire data was collected through the online survey program LimeSurvey (https://www.limesurvey.org/).

\section{Results}

Analyses were conducted using SPSS version 22.0 (IBM, 2013). Examination of the data indicated that the assumption of normality was violated for several variables, and as such transformations were conducted. However, as transformations did not change the significance 
or interpretation of any analyses, only untransformed data is presented. Missing data was observed across variables. Little's Missing Completely at Random Test (MCAR; Little, 1988) was significant $\left(\chi^{2}(444)=499.86, p=.034\right)$, indicating that systematic failure to respond to one or more items occurred. The highest rate of missing data occurred for the demographic item relating to field of study, with most of the missing data occurring among the international student sample (51.67\% of international students non-responding, $14.19 \%$ of domestic students non-responding). It was hypothesized that this item may not have been worded clearly for participants whose first language was other than English. Although imputation was considered for this variable, as it was not intended for use in the primary hypotheses and because the rate of non-responding was differential between the groups, field of study was removed from subsequent analyses. Descriptive data for the variable is, however, contained in Table 1. Removal of the variable returned a non-significant result on the MCAR test $\left(\chi^{2}(444)=483.11, p=.097\right)$, indicating that remaining data was missing completely at random. Listwise deletion by analysis was used to handle remaining missing data.

\section{Preliminary Analyses}

Chi-square and t-tests were conducted to investigate whether the international and domestic student populations were significantly different on demographic variables, as displayed in Table 1. Compared to domestic students, international students were more likely to be male $\left(\chi^{2}(1, N=357)=13.46, p<.001, V=.20\right)$, younger $(t(236)=2.77, p=.01, d=$ $.03)$, have been studying at university for less time $\left(\chi^{2}(7, N=357)=41.60, p<.001, V=\right.$ $.34)$, and have no previous use of MHSs $\left(\chi^{2}(1, N=357)=51.37, p<.001, V=.39\right)$.

As the study contained a quasi-experimental independent variable (whereby random allocation to the two groups was not possible), it was not surprising that differences emerged between the two groups on key demographic variables. To control for the potential the 
influence of these demographic variables on the outcome variables, a series of hierarchical regressions were conducted. More specifically, demographic variables found to be nonequivalent between the international and domestic student samples were first entered into the model (Model 1). These included gender $(0=$ male, $1=$ female $)$, age, previous use of MHSs $(0=$ no, $1=$ yes $)$, and years at university (dummy coded $0-7)$. To determine whether group differences existed after accounting for sampling differences in the previous step, student group $(0=$ domestic, 1 = international $)$ was entered into Model 2 and difference in model fit examined, to indicate whether student status accounted for variance in the outcome variable above and beyond that of the demographic variables.

\section{Main Analyses}

Descriptive statistics for international and domestic students' scores on the measures of psychological distress, MHL, help-seeking attitudes, and help-seeking intentions are displayed in Table 1.

Psychological distress. As described above, hierarchical multiple regressions were conducted with psychological distress as the response variable. Model 1 (Table 2) accounted for a small but significant amount of variance in psychological distress (approximately 6\%). The addition of student group in Model 2 did not significantly improve the amount of variance explained by Model 1, indicating that psychological distress was approximately equivalent between the domestic and international students after demographic differences in samples were accounted for. Age and previous use of MHSs were significant individual predictors in the model, with younger age and having accessed MHSs in the past both predictive of higher psychological distress.

Mental health literacy. A hierarchical multiple regression was conducted to assess the influence of demographic variables and student group on MHL. Model 1 (Table 3) was significant, explaining 26\% of the variance in MHL. Model 2 accounted for significantly 
more variance ( $41 \%$ total) than Model 1, indicating that student group was a significant predictor of MHL after controlling for differences in demographic variables. In order of importance in the final model, being an international student, having no previous contact with MHSs, being at university for a less time, and being male were associated with poorer MHL. Age was a significant predictor in Model 1, but became non-significant in Model 2, indicating that at least some of the variance in the response variable originally accounted for by age was better explained by student group.

Help-seeking attitudes. The IAMHS total score was used as the response variable to indicate help-seeking attitudes. Model 1 (Table 4) was significant, and showed that $40 \%$ of the variance in help-seeking attitudes was explained by the demographic variables entered. Model 2 was also significant, indicating that international student status explained a significant amount of variance beyond that of the demographic variables ( $45 \%$ total). In the final model, the most important predictor was student group with international student status being associated with poorer help-seeking attitudes. Age and gender were also significant predictors in the final model, such that younger age and being male were also associated with poorer help-seeking attitudes.

Help-seeking intentions for emotional problems. Model 1 (Table 5) of the hierarchical regression for help-seeking intentions for emotional problems was significant ( $7 \%$ of variance explained). The addition of student group in Model 2 did not explain significantly more variance than that already explained by the demographic variables $(8 \%$ total). In the final model, previous use of MHSs and age were significant predictors, such that previous use and older age were associated with more positive help-seeking intentions for emotional problems.

Help-seeking intentions for suicidal thoughts. Model 1 (Table 5) explained a significant amount of variance in help-seeking intentions for suicidal thoughts (approximately 
7\%). Model 2 explained significantly more variance than Model 1 (8\% total), indicating that student group accounted for variance above and beyond that already accounted for by the demographic variables. Student group was the most important individual predictor, with international student status being associated with poorer help-seeking intentions for suicidal thoughts. The only other significant predictor in the final model was years at university, with greater years of study being associated with more positive help-seeking intentions for suicidal thoughts.

\section{Discussion}

Consistent with calls for more research examining mental health needs and service use among international students (Raunic and Xenos, 2008), the aim of the present study was to examine the constructs of psychological distress, MHL, and help-seeking attitudes and intentions among international and domestic students. The study was quasi-experimental in nature due to the non-random allocation of participants to groups (domestic or international). Unsurprisingly, demographic differences were found between the two student groups. However, consistent with our hypotheses, differences between the two student samples still emerged once the potential influence of these demographic differences had been controlled. Specifically, international students reported lower help-seeking intentions for suicidal thoughts, and greater barriers to help-seeking, including lower mental health literacy and less favourable attitudes towards seeking help.

\section{Psychological Distress}

Previous research examining psychological distress and wellbeing between international and domestic students has been mixed (Fritz, et al., 2008; Hechanova-Alampay, Beehr, Christiansen, \& Van Horn, 2002; Janca and Hetzer, 1992; Rajapaksa and Dundes, 2002). In the current sample, no differences were found between the two student groups in terms of psychological distress, consistent with other recent studies conducted in Australia 
(Khawaja and Dempsey, 2008; Stallman, 2010). Regardless of group membership, the students in the current study reported moderate levels of distress, which supports previous findings that $75 \%$ of the tertiary students assessed reported moderate levels of distress (Pierceall and Keim, 2007). It is possible that international students studying in Australia do not experience the same degree of emotional distress and concerns as international students studying in other countries. However, nearly $40 \%$ of the international students in our sample had been living in Australia for over one year, thus it is possible that a significant proportion may have adapted and acculturated adequately to the challenges associated with studying abroad. Research has indicated that stressors such as accommodation problems and education system differences tend to be resolved or adjusted to earlier in the transition to university (Khawaja and Dempsey, 2008). Finally, consistent with previous literature (e.g., Stallman, 2010), we also found that being of younger age and having previously accessed MHSs were associated with higher levels of psychological distress. Considered within the context of theories of acculturation, it is possible that the students in the present sample had either engaged in effective cognitive, social and behavioural, and affective adaptation processes, or that engagement in these processes were not predictive of their psychological distress.

\section{Attitudes and Mental Health Literacy}

Also consistent with previous research (Fan, 1999; Shea and Yeh, 2008), international students had lower levels of MHL than their domestic counterparts. MHL was also lower among males, students who had been studying for less time, and students who had not previously had contact with MHSs. However, there was a statistically significant difference between domestic and international students on MHL after controlling for these demographic variables, and student group was the strongest predictor of MHL. These results support the proposition that being an international student may be related to additional barriers to accessing appropriate mental health treatment ( $\mathrm{Lu}$, et al., 2013). It is therefore not surprising 
that international student status was the strongest predictor of poorer attitudes toward accessing mental health services after controlling for related demographic variables, including younger age and being male. These findings are consistent with previous research demonstrating that concerns about stigma and the usefulness of mental health treatment are common among international students (Khawaja and Dempsey, 2008; Lu, et al., 2013), as is underutilisation of support services on university campuses (Mori, 2000). Less positive helpseeking attitudes reported by the international students may be a result of these students being more likely to use avoidance and other passive coping strategies to deal with their distress (Khawaja and Dempsey, 2008; Leong and Lau, 2001). That is, although levels of distress were similar between the two groups, it may be that international students cope with this distress via different mechanisms.

\section{Help-seeking Intentions and Behaviour}

In addition to poorer help-seeking attitudes, international students also reported lower help-seeking intentions (via professional sources) for suicidal ideation. No differences were found between groups for help-seeking intentions for emotional problems. These results are partially consistent with previous literature that suggests lower help-seeking intentions and reluctance to seek help exists among international students ( $\mathrm{Lu}$, et al., 2013). It has also been postulated that higher MHL levels and positive help-seeking attitudes are associated with higher help-seeking intentions (Smith and Shochet, 2011; Wilson, et al., 2005). In support of this notion, all three of these factors were found to be higher among domestic than international students. Years at university was also a significant predictor of helpseInternational students also reported less previous use of mental health services than domestic students. This finding should be considered within the context of the broader student mental health literature, which typically reports lower help-seeking and use of mental health services by university students, domestic and international, when compared to the 
general population (e.g., Eisenberg, et al., 2007). As such, international students may be considered to be a particularly vulnerable sub-group of a population with already low indicators of help-seeking intentions and behaviours.

\section{Limitations and Strengths}

Although the current research has provided increased understanding of international students and their needs, some limitations should be noted. First, all participants came from a single Australian institution, suggesting that they may not adequately represent the average tertiary student in Australia. This noted, use of one university also holds constant the university environment, context, and culture, thus enhancing the internal validity of the study. Second, even though some of the scales used in the study have been used previously in crosscultural settings (e.g. K-10), their validity on the basis of international students has not been evaluated. Furthermore, the relationship between field of study and the variables of interest was unable to be evaluated due to the high rate of missing data among international students for this item. Also, the group of international students were treated as a homogeneous group in the current study, as the purpose was to examine trends within the group as a whole. However, it is likely that differences exist among international students based on culture, gender, and other background and personal variables, which should be considered in future research. Lastly, although the design of the study allowed for a robust analysis by quantitative methods, utilising a mixed methods design would have enabled for a more in depth qualitative analysis to better understand the unique needs and difficulties experienced by this populations. Despite these limitations, it should be noted that the current study had several strengths. This study is one of only a few to assess the mental health literacy and helpseeking attitudes and intentions of international students, and in particular, to compare international students to a domestic student sample from the same study environment. 
Furthermore, the study was well powered and differences between groups were evident after controlling for existing demographic differences.

\section{Summary and Future Directions}

The comparison between the two student groups revealed that although both groups experience similar levels of psychological distress, the two groups differ on MHL levels, help-seeking attitudes, and help-seeking intentions for suicidal ideation. These findings have important implications for the tailoring of suitable interventions that focus on promoting the use of professional mental health services to students. International students may be in need of proactive interventions targeting mental health knowledge and help-seeking attitudes. Future research that includes the use of mixed or qualitative components may provide greater insight into the specific areas to target for interventions among tertiary international students. Such tailored interventions targeting mental health knowledge and attitudes may be beneficial in increasing student engagement with mental health services. These interventions would also provide financial benefit by reducing treatment costs through early intervention. In particular, future research may also be required to investigate the optimal timing and dosage of interventions throughout the acculturation process. Of particular interest for education providers, will be whether low intensity interventions, such as online and digital interventions, may be able to support international students through preventative approaches to improving mental health literacy and help-seeking intentions and attitudes. Such interventions have potential for wide and cost-effective dissemination, and may increase the likelihood of students' future engagement with mental health services, as needed. The development of tailored interventions for this population, digital or in person, will likely provide considerable benefit for international students experiencing mental health difficulties, an issue which is of particular importance given the exponential growth in international study and student populations. 


\section{References}

Adlaf, E., Gliksman, L., Demers, A., \& Newton-Taylor, B. (2001). The prevalence of elevated psychological distress among Canadian undergraduates: Findings from the 1998 Canadian campus survey. Journal of American College Health, 50, p 67.

Ang, P., \& Liamputtong, P. (2008). "Out of the circle": International students and the use of university counselling services. Australian Journal of Adult Learning, 48(1), pp. 109130.

Berry, J. W., Kim, U., Minde, T., \& Mok, D. (1987). Comparitive studies of acculturative stress. International Migration Review, 21(3), pp. 491-511.

Cooke, R., Bewick, B. M., Barkham, M., Bradley, M., \& Audin, K. (2006). Measuring, monitoring and managing the psychological well-being of first year university students. British Journal of Guidance \& Counselling, 34(4), pp. 505-517. doi:10.1080/03069880600942624

Deaux, K. (1996). Social identification.

Eisenberg, D., Golberstein, E., \& Gollust, S. E. (2007). Help-seeking and access to mental health care in a university student population. Medical care, 45(7), pp. 594-601.

Fan, C. (1999). A comparison of attitudes towards mental illness and knowledge of mental health services between Australian and Asian students Community Mental Health Journal, 35(1), pp. 47-57.

Faul, F., Erdfelder, E., Buchner, A., \& Lang, A. G. (2009). Statistical power analysis using G*Power 3.1: Tests for correlation and regression analyses Behaviour Research Methods, 41, pp. 1149-1160. doi:10.3758/BRM.41.4.1149

Fritz, M. V., Chin, D., \& DeMarinis, V. (2008). Stressors, anxiety, acculturation and adjustment among international and North American students. International Journal of Intercultural Relations, 32(3), pp. 244-259. 
Furnham, A., Cook, R., Martin, N., \& Batey, M. (2011). Mental health literacy among university students. Journal of Public Mental Health, 10(4), pp. 198-210. doi:10.1108/17465721111188223

Hechanova-Alampay, R., Beehr, T. A., Christiansen, N., \& Van Horn, R. K. (2002). Adjustment and strain among domestic and international student sojourners School Psychology International, 23(4), pp. 458-474. doi:10.1177/0143034302234007

Hunt, J., \& Eisenberg, D. (2010). Mental health problems and help-seeking behavior among college students. J Adolesc Health, 46(1), pp. 3-10. doi:10.1016/j.jadohealth.2009.08.008 Retrieved from http://www.ncbi.nlm.nih.gov/pubmed/20123251

IBM. (2013). IBM Statistics for Windows (Version Version 22.0). Armonk, NY IBM Corp Janca, A., \& Hetzer, J. E. (1992). Psychiatric morbidity of foreign students in Yugoslavia. International Journal of Social Psychiatry, 38, pp. 287-292.

Jorm, A. F., Korten, A. E., Jacomb, P., Christensen, H., Rodgers, B., \& Pollitt, P. (1997). "Mental health literacy": A survey of the public's ability to recognise mental disorders and their beliefs about the effectiveness of treatment. Medical Journal of Australia, 166, pp. 182-186.

Kessler, R. C., Barker, P. R., Colpe, L. J., Epstein, J. F., Gfroerer, J. C., Hiripi, E., \& Zaslavsky, A. M. (2003). Screening for serious mental illness in the general population Arch General Psychiatry, 60(2), pp. 184-189. doi:10.1017/S0033291702006074

Khawaja, N., \& Dempsey, J. (2007). Psychological distress in international university students: An Australian study Australian Journal of Guidance and Counselling, 17(1), pp. 13-27. 
Khawaja, N., \& Dempsey, J. (2008). A comparison of international and domestic tertiary students in Australia Australian Journal of Guidance and Counselling, 18(1), pp. 3046.

Khawaja, N., \& Stallman, H. M. (2011). Understanding the Coping Strategies of International Students: A Qualitative Approach. Australian Journal of Guidance and Counselling, 21(2), pp. 203-224. doi:10.1375/ajgc.21.2.203

Leong, F., \& Lau, A. (2001). Barriers to providing effective mental health services to Asian Americans Mental Health Services Research, 3(4), pp. 201-214.

Little, R. J. (1988). A test of missing completely at random for multivariate data with missing values. Journal of the American Statistical Association, 83(404), pp. 1198-1202.

Lu, S. H., Dear, B. F., Johnston, L., Wootton, B. M., \& Titov, N. (2013). An internet survey of emotional health, treatment seeking and barriers to accessing mental health treatment among Chinese-speaking international students in Australia. Counselling Psychology Quarterly, 27(1), pp. 96-108. doi:10.1080/09515070.2013.824408

Mackenzie, C. S., Knox, V., Gekoski, W., \& Macaulay, H. (2004). An adaptation and extension of the attitudes toward seeking professional psychological help scale. Journal of Applied Social Psychology, 34, pp. 2410-2435.

Morgan, T., Ness, D., \& Robinson, M. (2003). Student's help-seeking behaviours by gender, racial background, and student status Canadian Journal of Counselling, 37(2), pp. 151-166.

Mori, S. (2000). Addressing the mental health concerns of international students Journal of Counselling and Development, 78, pp. 17-24.

Nilsson, J. E., Berkel, L. A., Flores, L. Y., \& Lucas, M. S. (2004). Utilization Rate and Presenting Concerns of International Students at a University Counseling Center. 
Journal of College Student Psychotherapy, 19(2), pp. 49-59. doi:10.1300/J035v19n02_05

O’Connor, M., \& Casey, L. (2015). The mental health literacy scale (MHLS): A new scalebased measure of mental health literacy. Psychiatry Researchdoi:10.1016/j.psychres.2015.05.064

O’Connor, M., Casey, L., \& Clough, B. (2014). Measuring mental health literacy-a review of scale-based measures. Journal of mental health, 23(4), pp. 197-204.

Palmer, S., \& Puri, A. (2006). Coping with stress at university: A survival guide London Sage

Pierceall, E. A., \& Keim, M. C. (2007). Stress and Coping Strategies Among Community College Students. Community College Journal of Research and Practice, 31(9), pp. 703-712. doi:10.1080/10668920600866579

Rajapaksa, S., \& Dundes, L. (2002). Its a long way home: International student adjustment to living in the United States Journal of College Student Retention, 4(1), pp. 15-29.

Raunic, A., \& Xenos, S. (2008). University counselling service utilisation by local and international students and user characteristics: A review International Journal of Advanced Counselling, 30, pp. 262-267.

Reavley, N., \& Jorm, A. F. (2010). Prevention and early intervention to improve mental health in higher education students: a review. Early Interv Psychiatry, 4(2), pp. 132142. doi:10.1111/j.1751-7893.2010.00167.x Retrieved from

\section{http://www.ncbi.nlm.nih.gov/pubmed/20536969}

Reavley, N., McCann, T. V., \& Jorm, A. F. (2012). Mental health literacy in higher education students. Early Interv Psychiatry, 6(1), pp. 45-52. doi:10.1111/j.17517893.2011.00314.x Retrieved from http://www.ncbi.nlm.nih.gov/pubmed/22182365 
Ryan, M., Shochet, I., \& Stallman, H. M. (2010). Universal online interventions might engage psychologically distressed university students who are unlikely to seek formal help Advances in Mental Health, 9(1), pp. 73-83.

Sandhu, D. S. (1995). An examination of the psychological needs of the international students: Implications for counseling and psychotherapy. International Journal of Advanced Counselling, 17, pp. 229-239.

Sawir, E., Marginson, S., Deumert, A., Nyland, C., \& Ramia, G. (2008). Loneliness and international students: An Australian study. Journal of studies in international education, 12(2), pp. 148-180.

Shea, M., \& Yeh, C. (2008). Asian American students' cultural values, stigma, and relational self-construal: Correlates of attitudes toward professional help seeking. Journal of Mental Health Counseling, 30, pp. 157-172.

Smith, C. L., \& Shochet, I. M. (2011). The impact of mental health literacy on help-seeking intentions: results of a pilot study with first year psychology students. International Journal of Mental Health Promotion, 13(2), pp. 14-20.

Stallman, H. (2010). Psychological distress in university students: A comparison with general population data. Australian Psychologist, 45(4), pp. 249-257.

doi:10.1080/00050067.2010.482109

Stallman, H., \& Shochet, I. A. N. (2009). Prevalence of mental health problems in Australian university health services. Australian Psychologist, 44(2), pp. 122-127. doi:10.1080/00050060902733727

Toyokawa, T., \& Toyokawa, N. (2002). Extracurricular activities and the adjustment of Asian international students: A study of Japanese students. International Journal of Intercultural Relations, 26, pp. 363-379. 
Tsang, E. (2001). Adjustment of mainland Chinese academics and students to Singapore International Journal of Intercultural Relations, 25, pp. 347-372.

Ward, L. (1967). Some observations on the underlying dynamics of conflict in a foreign student. Journal of College Health Association, 10, pp. 430-440.

Wilson, G., Deane, F. P., \& Ciarrochi, J. (2005). Measuring help-seeking intentions:

Properties of the general help-seeking questionnaire Canadian Journal of

Counselling, 39(1), pp. 15-28. Retrieved from http://cjc-

\section{rcc.ucalgary.ca/cjc/index.php/rcc}

Yakushko, O., Davidson, M., \& Sanford-Martens, T. C. (2008). Seeking help in a foreign land: International students' use patterns for a US university counseling centre Jounral of College Counselling, 11(1), pp. 6-19.

Zhou, Y., Jindal-Snape, D., Topping, K., \& Todman, J. (2008). Theoretical models of culture shock and adaptation in international students in higher education. Studies in higher education, 33(1), pp. 63-75.

Table 1

Descriptive Statistics for international and domestic students across demographic and psychological variables

\begin{tabular}{lll}
\hline & Domestic & International \\
\cline { 2 - 3 } & $\mathrm{M}(\mathrm{SD}) / \mathrm{n}(\%)$ & $\mathrm{M}(\mathrm{SD}) / \mathrm{n}(\%)$ \\
\hline Age & $25.34(9.32)$ & $23.02(5.41)$ \\
Gender & & \\
Male & $30(19.1 \%)$ & $75(37.5 \%)$ \\
Female & $127(80.9 \%)$ & $125(62.5 \%)$ \\
Years at university & & \\
None & - & $34(17.0 \%)$ \\
Currently in first year & $60(38.2 \%)$ & $78(39.0 \%)$ \\
Currently in second year & $24(15.3 \%)$ & $28(14.0 \%)$ \\
Currently in third year & $23(14.6 \%)$ & $18(9.0 \%)$ \\
Currently in fourth year & $18(11.5 \%)$ & $5(2.5 \%)$ \\
Completed bachelor's & $5(3.2 \%)$ & $4(2.0 \%)$ \\
Currently postgraduate & $25(15.9 \%)$ & $28(14.0 \%)$ \\
Completed postgraduate & $2(1.3 \%)$ & $5(2.5 \%)$ \\
Years in Australia & & $57(28.5 \%)$ \\
$<3$ months & - & $38(19.0 \%)$ \\
$3-6$ months & $1(0.6 \%)$ & $33(16.5 \%)$ \\
$6-12$ months & $3(1.9 \%)$ & $50(25.0 \%)$ \\
$1-3$ years & $153(97.5 \%)$ & $22(11.0 \%)$ \\
$>3$ years & $34(26.8 \%)$ & $13(12.9 \%)$ \\
Study Discipline & & \\
Psychology & & \\
& &
\end{tabular}




\begin{tabular}{lll} 
Other health-related & $26(20.5 \%)$ & $18(17.8 \%)$ \\
Other & $67(52.8 \%)$ & $70(69.3 \%)$ \\
Previous use of MHSs & & \\
Yes & $91(58.0 \%)$ & $41(20.5 \%)$ \\
No & $66(42.0 \%)$ & $159(79.5 \%)$ \\
Psychological distress & $20.71(8.03)$ & $19.33(6.91)$ \\
Mental health literacy & $132.41(13.12)$ & $113.12(15.54)$ \\
Help-seeking attitudes & $67.54(15.53)$ & $57.90(12.43)$ \\
Psychological openness & $21.10(6.02)$ & $16.21(5.93)$ \\
Help-seeking propensity & $24.12(5.31)$ & $21.43(5.91)$ \\
Indifference to stigma & $22.34(7.54)$ & $20.30(6.53)$ \\
Highest Help-seeking intentions (E) & $5.74(1.31)$ & $5.25(1.45)$ \\
Highest Help-seeking intentions (S) & $5.89(1.38)$ & $5.29(1.70)$ \\
\hline
\end{tabular}

Note. $\mathrm{E}=$ emotional problems; $\mathrm{S}=$ suicidal thoughts. 
Table 2

Hierarchical multiple regression of demographic variables and student group on psychological distress

\begin{tabular}{|c|c|c|c|c|c|c|c|c|}
\hline \multirow[t]{2}{*}{ Variable } & \multicolumn{4}{|c|}{ Model 1} & \multicolumn{4}{|c|}{ Model 2} \\
\hline & $\mathrm{B}$ & $\beta$ & $\mathrm{sr}^{2}$ & $p$ & $\mathrm{~B}$ & $\beta$ & $\mathrm{sr}^{2}$ & $p$ \\
\hline Gender: Female & 0.92 & 0.06 & $<.01$ & .305 & 0.85 & 0.05 & $<.01$ & .347 \\
\hline Age & -0.13 & -0.13 & .02 & .023 & -0.13 & -0.13 & .02 & .022 \\
\hline Previous MHS Use & 3.19 & 0.21 & .04 & $<.001$ & 3.02 & 0.20 & .03 & .001 \\
\hline Years at University & -0.01 & $<0.01$ & $<.01$ & .971 & -0.02 & -0.01 & $<.01$ & .935 \\
\hline Student Group: International & & & & & -.514 & -.03 & $<.01$ & .562 \\
\hline $\mathrm{R}^{2}$ & & .06 & & & & .06 & & \\
\hline $\mathrm{F} / \mathrm{df}$ & & 5.00 & 4,325 & .001 & & 4.06 & 5,324 & .001 \\
\hline$\Delta \mathrm{R}^{2}$ & & & & & & $<.01$ & & \\
\hline$\Delta \mathrm{F} / \mathrm{df}$ & & & & & & .34 & 1,324 & .562 \\
\hline
\end{tabular}


Table 3

Hierarchical multiple regression of demographic variables and student group on MHL

\begin{tabular}{|c|c|c|c|c|c|c|c|c|}
\hline \multirow[t]{2}{*}{ Variable } & \multicolumn{4}{|c|}{ Model 1} & \multicolumn{4}{|c|}{ Model 2} \\
\hline & B & $\beta$ & $\mathrm{sr}^{2}$ & $p$ & B & $\beta$ & $\mathrm{sr}^{2}$ & $p$ \\
\hline Gender: Female & 6.40 & 0.17 & .03 & .003 & 4.65 & 0.12 & .01 & .016 \\
\hline Age & 0.25 & 0.12 & .01 & .046 & 0.17 & 0.08 & .01 & .126 \\
\hline Previous MHS Use & 11.56 & 0.33 & .10 & $<.001$ & 6.37 & 0.18 & .03 & .001 \\
\hline Years at University & 1.45 & 0.17 & .03 & .003 & 1.37 & 0.16 & .02 & .002 \\
\hline Student Group: International & & & & & -14.85 & -.43 & .15 & $<.001$ \\
\hline $\mathrm{R}^{2}$ & & .26 & & & & .41 & & \\
\hline $\mathrm{F} / \mathrm{df}$ & & 22.55 & 4,257 & $<.001$ & & 36.09 & 5,256 & $<.001$ \\
\hline$\Delta \mathrm{R}^{2}$ & & & & & & .15 & & \\
\hline$\Delta \mathrm{F} / \mathrm{df}$ & & & & & & 67.07 & 1,256 & $<.001$ \\
\hline
\end{tabular}


Table 4

Hierarchical multiple regression of demographic variables and student group on Help-seeking Attitudes

\begin{tabular}{|c|c|c|c|c|c|c|c|c|}
\hline \multirow[t]{2}{*}{ Variable } & \multicolumn{4}{|c|}{ Model 1} & \multicolumn{4}{|c|}{ Model 2} \\
\hline & $\mathrm{B}$ & $\beta$ & $\mathrm{sr}^{2}$ & $p$ & $\mathrm{~B}$ & $\beta$ & $\mathrm{sr}^{2}$ & $p$ \\
\hline Gender: Female & 6.02 & 0.19 & .03 & .001 & 5.27 & 0.16 & .03 & .002 \\
\hline Age & 0.37 & 0.20 & .03 & .001 & 0.35 & 0.18 & .03 & .001 \\
\hline Previous MHS Use & 5.42 & 0.18 & .04 & .001 & 3.22 & 0.11 & .01 & .065 \\
\hline Years at University & 0.49 & 0.07 & $<.01$ & .240 & 0.41 & 0.07 & $<.01$ & .317 \\
\hline Student Group: International & & & & & -6.29 & -.21 & .04 & $<.001$ \\
\hline $\mathrm{R}^{2}$ & & .40 & & & & .45 & & \\
\hline $\mathrm{F} / \mathrm{df}$ & & 14.61 & 4,305 & $<.001$ & & 15.05 & 5,304 & $<.001$ \\
\hline$\Delta \mathrm{R}^{2}$ & & & & & & .04 & & \\
\hline$\Delta \mathrm{F} / \mathrm{df}$ & & & & & & 14.27 & 1,304 & $<.001$ \\
\hline
\end{tabular}


Table 5

Hierarchical multiple regression of demographic variables and student group on Help-seeking Intentions

\begin{tabular}{|c|c|c|c|c|c|c|c|c|c|}
\hline & \multirow[t]{2}{*}{ Variable } & \multicolumn{4}{|c|}{ Model 1} & \multicolumn{4}{|c|}{ Model 2} \\
\hline & & B & $\beta$ & $\mathrm{sr}^{2}$ & $p$ & B & $\beta$ & $\mathrm{sr}^{2}$ & $p$ \\
\hline \multirow{9}{*}{ 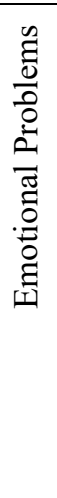 } & Gender: Female & $<0.01$ & $<0.01$ & $<.01$ & .988 & -0.03 & -0.01 & $<.01$ & .869 \\
\hline & Age & 0.03 & 0.15 & .02 & .020 & 0.03 & 0.15 & .02 & .027 \\
\hline & Previous MHS Use & 0.53 & 0.19 & .03 & .004 & 0.44 & 0.16 & .02 & .025 \\
\hline & Years at University & 0.01 & 0.01 & $<.01$ & .842 & 0.01 & 0.01 & $<.01$ & .859 \\
\hline & Student Group: Int & & & & & -0.26 & -0.09 & $<.01$ & .158 \\
\hline & $\mathrm{R}^{2}$ & & .07 & & & & .08 & & \\
\hline & $\mathrm{F} / \mathrm{df}$ & & 5.00 & 4,254 & .001 & & 4.41 & 5,253 & .001 \\
\hline & $\Delta \mathrm{R}^{2}$ & & & & & & .01 & & \\
\hline & $\Delta \mathrm{F} / \mathrm{df}$ & & & & & & 2.00 & 1,253 & .158 \\
\hline \multirow{9}{*}{ 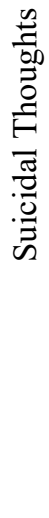 } & Gender: Female & -0.23 & -0.07 & $<.01$ & .292 & -0.28 & -0.08 & .01 & .203 \\
\hline & Age & 0.02 & 0.08 & .01 & .224 & 0.01 & 0.07 & $<.01$ & .295 \\
\hline & Previous MHS Use & 0.49 & 0.15 & .02 & .018 & 0.33 & 0.11 & .01 & .124 \\
\hline & Years at University & 0.11 & 0.14 & .02 & .037 & 0.10 & 0.13 & .02 & .039 \\
\hline & Student Group: Int & & & & & -0.44 & -0.14 & .02 & .037 \\
\hline & $\mathrm{R}^{2}$ & & .07 & & & & .08 & & \\
\hline & $\mathrm{F} / \mathrm{df}$ & & 4.48 & 4,254 & .002 & & 4.51 & 5,253 & .001 \\
\hline & $\Delta \mathrm{R}^{2}$ & & & & & & .02 & & \\
\hline & $\Delta \mathrm{F} / \mathrm{df}$ & & & & & & 4.38 & 1,253 & .037 \\
\hline
\end{tabular}

